\title{
Plasma Metabolites and Risk of Radiation-induced Esophagitis: A Secondary Analysis from a Prospective Study
}

\author{
DANIEL J. TANDBERG ${ }^{1}$, TRACY HOLT ${ }^{2}$ and CHRIS R. KELSEY ${ }^{1}$ \\ ${ }^{1}$ Department of Radiation Oncology, Duke University School of Medicine, Durham, NC, U.S.A.; \\ ${ }^{2}$ Metabolon, Inc., Durham, NC, U.S.A.
}

\begin{abstract}
Aim: Metabolic profiling was performed on plasma samples obtained prior to and during radiation therapy $(R T)$ for locally advanced lung cancer to identify metabolites predictive of RT-induced esophagitis. Patients and Methods: Patients received cisplatin/etoposide with RT as part of a prospective dose-escalation study $(n=24)$. Plasma samples were collected at baseline, weeks 2 and 5 during $R T$, and 6 weeks post-RT. Metabolites were measured by ultrahigh-performance liquid chromatography-tandem mass spectroscopy at each time-point. Metabolite concentrations were compared between patients developing grade 0-1 and those with grade 2 or more esophagitis. Results: At baseline, 23 metabolites differed significantly $(p<0.05)$ between patients with grade 0-1 esophagitis and those with grade 2 or esophagitis. Sixty-seven metabolites were different at week 2. None reached statistical significance $(q<0.05)$ after corrections for multiple comparisons. On random forest modeling, the predictive accuracy of the metabolite data was $33 \%$ at baseline and $50 \%$ at 2 weeks. Conclusion: No individual metabolite or group of metabolites was predictive of acute RT-induced esophagitis.
\end{abstract}

The most effective treatment approach for locally advanced lung cancer, whether non-small cell (NSCLC) or small cell, is concurrent chemotherapy and radiation therapy (RT). RTinduced esophagitis is a primary dose-limiting acute toxicity of concurrent chemotherapy and RT. Severe esophagitis can result in significant morbidity during treatment and inferior clinical outcomes $(1,2)$.

This article is freely accessible online.

Correspondence to: Daniel J. Tandberg, MD, Department of Radiation Oncology, Duke University School of Medicine, DUMC Box 3085, Durham, NC 27710, U.S.A. Tel: +1 9196687336, Fax: +19196687345, e-mail: daniel.tandberg@duke.edu

Key Words: Lung cancer, esophagitis, biomarkers, metabolomics, radiation therapy.
Numerous clinical and dosimetric factors have been associated with increasing risk of RT-induced esophagitis. Clinical factors include nodal stage of $\mathrm{N} 2$ or worse, hyperfractioned RT, pre-existing dysphagia, and concurrent chemotherapy $(3,4)$. Dosimetric factors include increasing mean esophagus dose, maximum esophageal dose, as well as a wide range of volumetric parameters [e.g. esophageal volume receiving >35 Gy (V35), V45, V50, and V60] (3-6). However, a single best threshold volumetric predictor of high-grade esophagitis has not been identified (6). Thus, there is a need for alternative predictors of esophagitis, such as biological biomarkers, to assist in the accurate prediction of esophagitis. By identifying patients at increased risk of high-grade RT-induced esophagitis, modifications in RT technique (e.g. intensity-modulated RT, image guidance) or aggressive and early supportive care could be utilized to minimize the risk of, or complications from, esophagitis.

Metabolomics refers to the identification and study of small-molecule chemicals which are the end-products of cellular processes. Alterations in genetic, transcriptional, and proteomic processes, as well as lifestyle/environmental changes can result in changes in the metabolome. There is interest in utilizing metabolomics to identify biomarkers predictive of RT response and toxicity (7). Indeed, studies in mice have identified metabolites correlated with RT-induced damage to the intestinal tract and lung $(8,9)$. To our knowledge, evaluation of metabolites predictive of RTinduced esophagitis has not been reported in a human population. Thus, we performed metabolic profiling of prospectively collected plasma samples from patients receiving concurrent chemotherapy and RT for locally advanced lung cancer to determine if concentration differences of single metabolites or panels of metabolites could be predictive of high-grade RT-induced esophagitis.

\section{Patients and Methods}

As part of an Institutional Review Board-approved prospective clinical trial (Clinicaltrials.gov identifier NCT00921739), patients with locally advanced lung cancer were treated with escalating doses of accelerated RT with concurrent chemotherapy (10). All patients 
underwent RT planning with custom immobilization, esophageal and intravenous contrast, and 4-dimensional computed tomographic (CT) imaging to assess volumes throughout the respiratory cycle. Intensitymodulated RT planning was used in all cases. RT was administered using six fractions per week (two fractions on Fridays with a 6-hour minimum interval between fractions). Daily image guidance using onboard imaging supplemented with weekly cone-beam CT scans was utilized. The total dose of RT was escalated from 58 Gy to $74 \mathrm{~Gy}$. Patients received concurrent chemotherapy with cisplatin $\left(50 \mathrm{mg} / \mathrm{m}^{2}\right.$ on days $1,8,29$, and 36 ) and etoposide $\left(50 \mathrm{mg} / \mathrm{m}^{2}\right.$ on days $1-5$ and 29-33). Toxicity, including RT-induced esophagitis, was recorded at weekly clinic appointments during RT and at 6 weeks post-RT using Common Terminology Criteria for Adverse Events version 4.03 (11). In addition, non-fasting plasma samples were collected at baseline, weeks 2 and 5 during RT, and 6 weeks post-RT per study protocol. Samples were immediately stored at $-80^{\circ} \mathrm{C}$.

Metabolite assessment. Metabolic profiling of plasma samples from all time points was performed by Metabolon, Inc. (Durham, NC) (12). Briefly, samples were given a unique identifier and then transferred to Metabolon on dry ice and subsequently stored at $-80^{\circ} \mathrm{C}$ prior to processing. Proteins were precipitated from each sample with methanol. Metabolite profiling was performed using ultrahigh-performance liquid chromatography-tandem mass spectroscopy (UPLC-MS/MS). UPLC-MS/MS was performed using the Waters ACQUITY ultra-performance liquid chromatography system (Waters Corporation, Milford, MA, USA) and a Thermo Scientific Q-Exactive highesolution/accurate mass spectrometer (Thermo Fisher Scientific Inc., Waltham, MA, USA) interfaced with a heated electrospray ionization source and Orbitrap mass analyzer (Thermo Fisher Scientific Inc.) operated at 35,000 mass resolution. Compounds were identified by comparison to more than 4,500 library entries of purified standards which included data on retention time/index, mass to charge ratio, and chromatographic data (including MS/MS spectral data). Metabolite quantification was performed by calculation of the area under the curve of the peaks. A total of 760 metabolites were identified and analyzed. Pathways included amino acid $(n=187)$, peptide $(n=21)$, carbohydrate $(n=24)$, energy $(n=9)$, lipid $(n=287)$, nucleotide $(n=32)$, cofactors and vitamins $(n=36)$, and xenobiotics $(n=164)$.

Controls were analyzed in concert with the experimental samples for quality assurance. A pooled matrix sample generated by taking a small volume of each experimental sample served as a technical replicate throughout the data set. Extracted water samples served as process blanks. Finally, a cocktail of quality control standards were spiked into every analyzed sample for instrument performance monitoring and to aide chromatographic alignment.

Statistical analysis. The primary objective of this biological correlative analysis was to identify biological metabolites predictive of RT-induced esophagitis that could be detected before or early (week 2) within a course of RT.

Patients were placed into cohorts based on the maximum grade of esophagitis developed during or within 6 weeks of RT (esophagitis grade $0-1$ and grade 2 or more). The Fisher exact and Mann-Whitney $U$-tests were used to compare patient and treatment characteristics between cohorts. Mixed effects ANOVA contrasts were used to compare individual metabolite concentrations between the two groups at each study time point. Correlation analysis was performed comparing the relationship between observed changes in
Table I. Patient characteristics, $N=24$.

\begin{tabular}{ll}
\hline Characteristic & \\
\hline Median age (range), years & $64(49-74)$ \\
Gender, n (\%) & $12(50)$ \\
$\quad$ Female & $12(50)$ \\
$\quad$ Male & \\
Histology, n (\%) & $21(88)$ \\
$\quad$ Non-small cell & $3(12)$ \\
Small cell & \\
Stage, n (\%) & $8(33)$ \\
$\quad$ IIIA & $10(42)$ \\
IIIB & $6(25)$ \\
Recurrent (N2 or N3)
\end{tabular}

metabolite levels and mean esophageal dose at each time point and all time points combined. An estimate of the false-discovery rate ( $q$ value) was calculated to account for multiple comparisons.

Random forest analysis was performed to assess the predictive accuracy of the metabolite dataset in distinguishing esophagitis class (13). This was performed at each of the study time points. A classification error rate (out-of-bag error rate) was determined by comparing the class predictions based on the random forest, using leave-one-out cross validation, to the true classes. To determine which metabolites made the largest contribution to the group classification, a variable importance measure termed 'mean decrease accuracy' was computed by randomly permuting a metabolite, running the observed values through the trees, and then reassessing the prediction accuracy. For metabolites not important to group classification, this procedure would result in little change in the accuracy of the class prediction. This process was used to create an importance rank ordering of metabolites based on the random forest analysis.

Statistical analysis was performed with Array Studio (Omic Soft Corporation, Cary, NC) and R statistical software. $p$-Values of less than 0.05 were considered statistically significant. After correction for multiple comparisons, a $q$-value of less than 0.05 was considered significant, consistent with less than a $5 \%$ false-discovery rate.

\section{Results}

Between 2009 and 2014, 24 patients were enrolled onto this phase I study. The majority of patients had NSCLC (88\%). Patient characteristics can be found in Table I. The median cumulative RT dose was 68 Gy (range=58-74 Gy). All patients received concurrent cisplatin and etoposide chemotherapy and this was administered as planned in $23 / 24$ patients $(96 \%)$. Of 96 potential plasma samples, 90 were obtained per protocol, while six were not, all due to patient refusal.

The median esophageal V20 and V60 values were 34\% $($ range $=21-65 \%)$ and $5 \%$ (range $=0-41 \%)$, respectively. The mean esophageal dose was $20 \mathrm{~Gy}$ (range=8-38 Gy). Ten patients $(42 \%)$ developed acute grade $0-1$ esophagitis and 14 patients $(58 \%)$ grade $2-3$ esophagitis. There were no cases of acute grade 4 or 5 esophagitis. There were no significant 
Table II. Number of individual metabolites with significant differences in concentration between grade 0-1 and grade 2 or more radiotherapy (RT)induced esophagitis at each study time-point.

\begin{tabular}{lcccc}
\hline & \multicolumn{3}{c}{ Time point } \\
\cline { 2 - 5 } & Baseline & 2 Weeks RT & 5 Weeks RT & 6 Weeks post RT \\
\hline Total number of metabolites (at $p<0.05)$ & 23 & 67 & 20 & 17 \\
Change in metabolite concentration $(\uparrow \mid \downarrow)^{*}$ & 13 । 10 & 6 । 61 & 7 । 13 & 4 । 13 \\
Total number of metabolites $(q<0.05)^{* *}$ & 0 & 0 & 0 & 0 \\
\hline
\end{tabular}

*Number of metabolites significantly $(p<0.05)$ increased $(\uparrow)$ or decreased $(\downarrow)$ in concentration in esophagitis grade 2 or more compared to grade $0-1$. **Total number of metabolites significantly different after correction for multiple comparisons, false-discovery rate $<5 \%$.

Table III. Number of individual metabolites with significant differences in concentration at each study time point compared to baseline.

\begin{tabular}{|c|c|c|c|c|c|c|}
\hline & \multicolumn{3}{|c|}{ Esophagitis grade $\geq 2$} & \multicolumn{3}{|c|}{ Esophagitis grade $0-1$} \\
\hline & 2 Weeks RT & 5 Weeks RT & 6 Weeks post RT & 2 Weeks RT & 5 Weeks RT & 6 Weeks post RT \\
\hline Total number of metabolites (at $p<0.05$ ) & 252 & 181 & 86 & 145 & 119 & 61 \\
\hline Change in metabolites $(\uparrow I \downarrow)^{*}$ & $102 \mid 150$ & $101 \mid 80$ & 49 | 37 & $93 \mid 52$ & $76 \mid 43$ & $50 \mid 11$ \\
\hline Total number of metabolites $(q<0.05)^{* *}$ & 201 & 112 & 4 & 31 & 19 & 1 \\
\hline
\end{tabular}

*Number of metabolites significantly $(p<0.05)$ increased $(\uparrow)$ or decreased $(\downarrow)$ in concentration at study time point compared to baseline. **Total number of metabolites significantly different after correction for multiple comparisons, false-discovery rate $<5 \%$.

differences in esophageal V20 values (37\% vs. 36\%, $p=0.83)$, V60 values ( $6 \%$ vs. $10 \%, p=0.24)$, or mean esophageal doses ( $19 \mathrm{~Gy} v s .20 \mathrm{~Gy}, p=0.65$ ) between the cohorts with grade 01 and grade 2 or more esophagitis, respectively.

A total of 760 metabolites were identified. At baseline, the concentration of 23 individual metabolites differed significantly $(p<0.05)$ between the cohorts of patients who developed grade $0-1$ esophagitis versus those developing grade 2 or more esophagitis. However, after correction for multiple comparisons, there were no statistically significant differences. At 2 weeks, there were 67 metabolites with significant differences between the esophagitis cohorts, 20 at week 5, and 17 at 6 weeks post-RT. Once again, none remained statistically significant after correction for multiple comparisons (Table II).

Next we performed random foresting modeling using metabolite concentrations to differentiate the two cohorts. At the baseline time-point, the random forest predictive accuracy (ability to accurately differentiate between grade $0-1$ and grade 2 or more esophagitis) using all metabolite data was $33 \%$. The 30 metabolites with the highest mean decrease accuracy values (measure of group differentiation contribution) are shown in Figure 1a. Specific metabolites with the greatest discriminatory potential at baseline were 1-linoleoylglycerophosphatidic acid (18:2), $\mathrm{N}$-acetylglutamate, and trans4-hydroxyproline. At 2 weeks, the random forest predictive accuracy was $50 \%$ with 3-hydroxydecanoate, $\mathrm{N}$-methylproline, and stearoyl ethanolamine being most discriminatory (Figure 1b). The three most discriminatory metabolites at baseline and 2 weeks were all lower in concentration in patients with grade 2 or more esophagitis compared to grade $0-1$.

We assessed changes in the concentration of individual metabolites during treatment by comparing the plasma metabolite concentrations at each study time point to their baseline concentrations. After correction for multiple comparisons, there were significant changes in individual metabolite concentrations during radiotherapy, with a greater number of significant changes in patients with grade 2 or more esophagitis compared to patients with grade 0-1 esophagitis. In both esophagitis cohorts, the largest number of significant changes was seen at week 2 of RT (Table III).

Finally, we investigated whether cumulative radiation exposure to the esophagus was correlated with change in individual metabolite levels. After baseline normalization, 57 metabolites were significantly correlated to the mean esophageal dose near the end of RT $(p<0.05)$. After correction for multiple comparisons, ribitol (a carbohydrate in pentose metabolism) was the only metabolite that maintained significance $(q<0.05)$.

\section{Discussion}

In this exploratory analysis, we attempted to identify plasma metabolites worthy of further investigation as biomarkers predictive of RT-induced esophagitis in patients receiving 


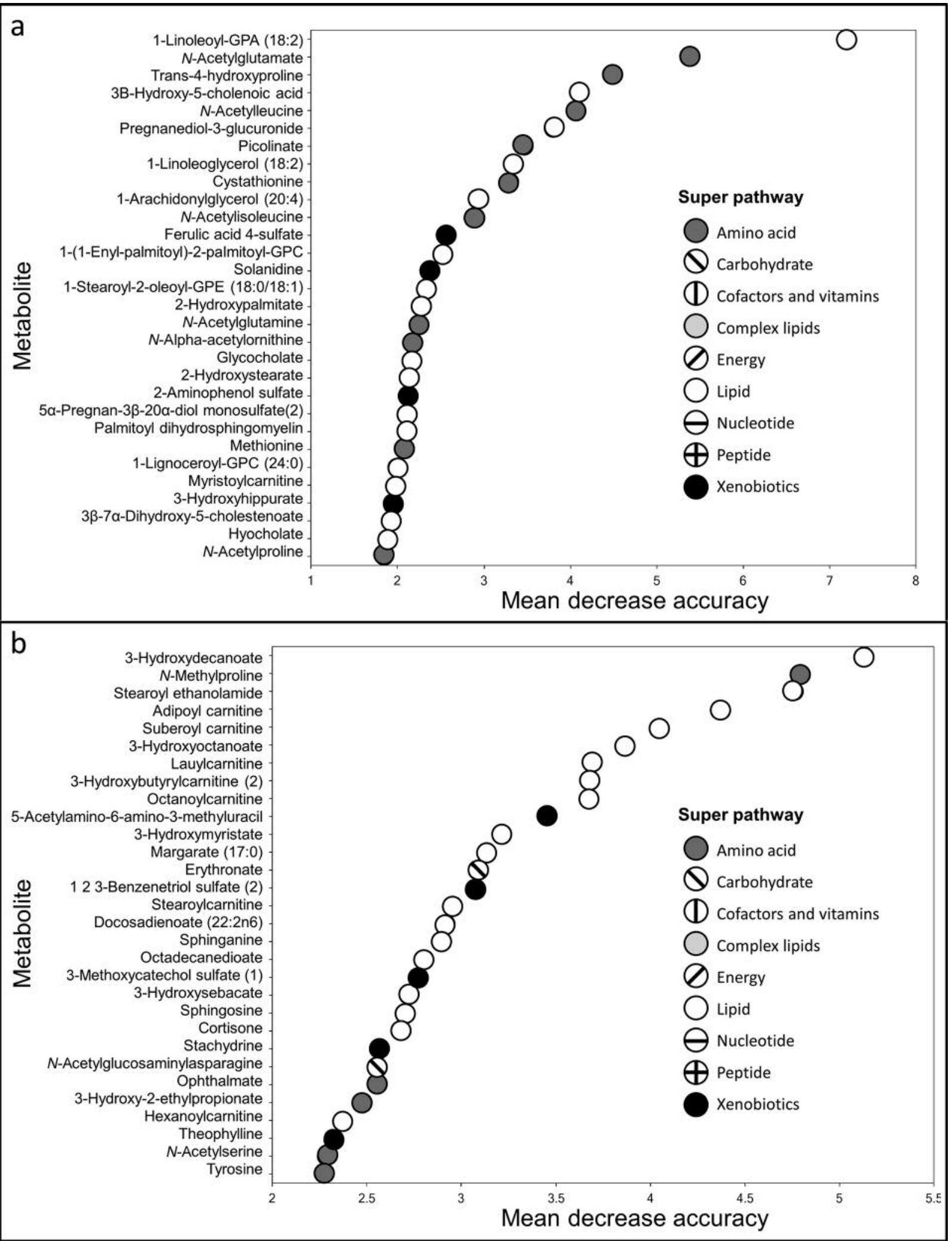

Figure 1. Graph of individual metabolites most important for differentiating patients with grade 0-1 radiotherapy (RT)-induced esophagitis from those with grade 2 or more in the random forest analysis using baseline plasma samples (a) and plasma samples from week 2 of RT $(b)$. Metabolites with higher mean decrease accuracy contribute more to group differentiation. 
concurrent chemotherapy and RT for locally advanced lung cancer. Metabolic profiling of plasma samples obtained prior to, during, and after treatment was performed. Metabolite concentrations in patients who developed grade 0-1 esophagitis were compared to patients who developed grade 2 or more esophagitis. Overall, we found no association between plasma metabolites and RT-induced esophagitis. At baseline and shortly after initiation of treatment (week 2), we did not identify any metabolites that were significantly different between patients with grade $0-1$ esophagitis and those with grade 2 or more esophagitis after correction for multiple testing. Models based on differences in metabolite concentration were not able to discriminate with good accuracy individuals who developed high-grade esophagitis from those who did not.

RT-induced esophagitis is a common acute side-effect of concurrent chemotherapy and RT for locally advanced lung cancer. Numerous clinical and dosimetric variables have been associated with risk of developing esophagitis (3-6). Data regarding biological predictors of esophagitis is more limited. Potential genetic predictors of RT-induced esophagitis including microRNA-related genetic variations (14) single nucleotide polymorphisms in inflammation-related genes (15), genotype of the transforming growth factor beta-1 (TGF $\beta 1$ ) gene (16), and TGFB1 and xeroderma pigmentosum group $\mathrm{D}(X P D)$ single nucleotide polymorphisms (17) have been reported. To the best of our knowledge, metabolites have not been explored as potential biomarkers of RT-induced esophagitis, which prompted this study.

Metabolites are promising potential biomarkers for disease/toxicity as they are felt to be representative of the current physiology of the organism (7). Furthermore, technological advances have provided the means to accurately and rapidly perform high-throughput metabolomics analyses (18). Several studies have used metabolomics to identify differences in the metabolic profile of patients with lung cancer compared to controls $(19,20)$. Studies utilizing metabolite data to predict RT-induced toxicity are more limited. In a murine model, Ghosh et al. demonstrated the potential utility of metabolomics in predicting RT toxicity. In mice exposed to whole-body RT, they were able to identify RT-mediated metabolite markers of gastrointestinal tract injury (9). In human patients receiving pelvic RT, plasma levels of the amino acid metabolite citrulline have been correlated to intestinal toxicity (21). Finally, supplementation with glutamine, an amino acid metabolite, has been associated with decreased rates of high-grade esophagitis in patients receiving RT for lung cancer $(22,23)$. Taken together, these studies suggest a possible association between metabolites and RT toxicity.

In our analysis, we did not identify an individual metabolite that was predictive of RT-induced esophagitis. After strict correction for multiple comparisons (false- discovery rate of $<5 \%$, there were no metabolites significantly different in concentration between patients with grade $0-1$ esophagitis and those with grade 2 or more esophagitis. This was the case at each study time point. However, uncorrected $p$-values did suggest differences in metabolites between esophagitis cohorts, most prominently at week 2 of RT. Of note, the metabolite glutamine, which has been explored as a potential supplement to prevent RTinduced esophagitis, was not significantly different between esophagitis cohorts at any of the study time points, even before correction for multiple comparisons. Citrulline was also not significantly different.

We observed significant changes in the serum metabolome during RT, with a greater number of changes among patients with grade 2 or more esophagitis. When compared to baseline, the number of significant changes in metabolite concentrations was greatest early after treatment initiation (2 weeks). Metabolic profiling with quantification of the number of changes to the metabolome shortly after treatment initiation could serve as a potential marker for RT-induced toxicity, and might be worthy of further analysis. However, while there were changes in the metabolome there were a limited number of individual metabolites whose concentration correlated to RT dose to the esophagus. This is possibly explained by metabolites increasing and decreasing during the course of RT.

It is unlikely that a single metabolite will be effective as a predictive biomarker of RT-induced toxicity. More likely, pooled data from a panel of metabolites will be necessary to discriminate esophagitis risk. Through random forest analysis, we assessed the predictive accuracy of all the metabolite data in differentiating grade 0-1 from grade 2 or more esophagitis. Metabolite data from baseline and 2 weeks did not prove to be effective at distinguishing cohorts, with predictive accuracies of only $33 \%$ and $50 \%$, respectively. Thus, it does not appear that global metabolic profiling performed prior to or early after initiation of RT is an effective means to identify patients who will develop highgrade esophagitis. Further research may be able to use these data to identify more selected panels of metabolites predictive of esophagitis.

To the best of our knowledge, this analysis is the first to use metabolic profiling to identify potential predictive biomarkers for RT-induced esophagitis. Strengths of this study include the prospective design and prospective collection of toxicity data. Moreover, we performed metabolic profiling at multiple time-points, which provides a more comprehensive picture of metabolic differences throughout the treatment course. Finally, we used a validated method of metabolite assessment with multiple control methods to ensure the instrument variability and overall process variability was up to standard. There are several important limitations to our study. Due to the small sample 
size coupled with the large number of metabolites analyzed, it is possible that our findings could be due to chance. Furthermore, potentially strong associations may be missed after correction for multiple comparisons. Finally, there are many variables that could impact metabolite concentrations, including diet and time of collection, and these were not controlled for.

In conclusion, this exploratory metabolomics analysis did not identify individual metabolites or groups of metabolites associated with risk of RT-induced esophagitis in patients receiving concurrent chemotherapy and RT for locally advanced lung cancer. Clinical, and especially dosimetric variables, currently remain the most valuable tools in predicting a patient's risks of developing high-grade RTinduced esophagitis.

\section{Conflict of Interests}

Tracy Holt is an employee of Metabolon, Inc.. Daniel Tandberg and Chris Kelsey have no conflicts of interest in relation to the subject matter.

\section{Acknowledgements}

This study was funded through a research grant from Varian Medical Systems (Palo Alto, CA, USA).

\section{References}

1 Cox JD, Pajak TF, Asbell S, Russell AH, Pederson J, Byhardt RW, Emami B and Roach M: Interruptions of high-dose radiation therapy decrease long-term survival of favorable patients with unresectable non-small cell carcinoma of the lung: Analysis of 1244 cases from 3 Radiation Therapy Oncology Group (RTOG) trials. Int J Radiat Oncol Biol Phys 27(3): 493498, 1993.

2 Bradley JD, Paulus R, Komaki R, Masters G, Blumenschein G, Schild S, Bogart J, Hu C, Forster K, Magliocco A, Kavadi V, Garces YI, Narayan S, Iyengar P, Robinson C, Wynn RB, Koprowski C, Meng J, Beitler J, Gaur R, Curran W and Choy $\mathrm{H}$ : Standard-dose versus high-dose conformal radiotherapy with concurrent and consolidation carboplatin plus paclitaxel with or without cetuximab for patients with stage IIIA or IIIB non-smallcell lung cancer (RTOG 0617): A randomised, two-by-two factorial phase 3 study. Lancet Oncol 16(2): 187-199, 2015.

3 Ahn SJ, Kahn D, Zhou S, Yu X, Hollis D, Shafman TD and Marks LB: Dosimetric and clinical predictors for radiationinduced esophageal injury. Int J Radiat Oncol Biol Phys 61(2): 335-347, 2005.

4 Singh AK, Lockett MA and Bradley JD: Predictors of radiationinduced esophageal toxicity in patients with non-small-cell lung cancer treated with three-dimensional conformal radiotherapy. Int J Radiat Oncol Biol Phys 55(2): 337-341, 2003.

5 Belderbos J, Heemsbergen W, Hoogeman M, Pengel K, Rossi M and Lebesque J: Acute esophageal toxicity in non-small cell lung cancer patients after high dose conformal radiotherapy. Radiother Oncol 75(2): 157-164, 2005.
6 Werner-Wasik M, Yorke E, Deasy J, Nam J and Marks LB: Radiation dose-volume effects in the esophagus. Int J Radiat Oncol Biol Phys 76(3 Suppl): S86-93, 2010.

7 Menon SS, Uppal M, Randhawa S, Cheema MS, Aghdam N, Usala RL, Ghosh SP, Cheema AK and Dritschilo A: Radiation metabolomics: Current status and future directions. Front Oncol 6: 20,2016

8 Jones JW, Scott AJ, Tudor G, Xu PT, Jackson IL, Vujaskovic Z, Booth C, MacVittie TJ, Ernst RK and Kane MA: Identification and quantitation of biomarkers for radiationinduced injury via mass spectrometry. Health Phys 106(1): 106-119, 2014.

9 Ghosh SP, Singh R, Chakraborty K, Kulkarni S, Uppal A, Luo Y, Kaur P, Pathak R, Kumar KS, Hauer-Jensen M and Cheema AK: Metabolomic changes in gastrointestinal tissues after whole-body radiation in a murine model. Mol Biosyst 9(4): 723731, 2013.

10 Kelsey CR, Das S, Gu L, Dunphy FR, Ready NE and Marks LB: Phase 1 dose escalation study of accelerated radiation therapy with concurrent chemotherapy for locally advanced lung cancer. Int J Radiat Oncol Biol Phys 93(5): 997-1004, 2015.

11 National Cancer Institute, National Institutes of Health (2010). Common Terminology Criteria for Adverse Events v4.03, [online]. Available: https://evs.nci.nih.gov/ftp1/CTCAE/ CTCAE_4.03_2010-06-14_QuickReference_8.5x11.pdf (last accessed July 11, 2016).

12 Evans AM, DeHaven CD, Barrett T, Mitchell M and Milgram E: Integrated, nontargeted ultrahigh performance liquid chromatography/electrospray ionization tandem mass spectrometry platform for the identification and relative quantification of the smallmolecule complement of biological systems. Anal Chem 81(16): 6656-6667, 2009.

13 Brieman L: Random forests. Machine Learning 45(1): 5-32, 2001.

14 Li R, Pu X, Chang JY, Ye Y, Komaki R, Minna JD, Roth JA, Han B and Wu X: Mirna-related genetic variations associated with radiotherapy-induced toxicities in patients with locally advanced non-small cell lung cancer. PLoS One 11(3): $\mathrm{e} 0150467,2016$.

15 Hildebrandt MA, Komaki R, Liao Z, Gu J, Chang JY, Ye Y, Lu C, Stewart DJ, Minna JD, Roth JA, Lippman SM, Cox JD, Hong WK, Spitz MR and Wu X: Genetic variants in inflammationrelated genes are associated with radiation-induced toxicity following treatment for non-small cell lung cancer. PLoS One 5(8): e12402, 2010.

16 Guerra JL, Gomez D, Wei Q, Liu Z, Wang LE, Yuan X, Zhuang Y, Komaki R and Liao Z: Association between single nucleotide polymorphisms of the transforming growth factor $\beta 1$ gene and the risk of severe radiation esophagitis in patients with lung cancer. Radiother Oncol 105(3): 299-304, 2012.

17 Zhang L, Yang M, Bi N, Ji W, Wu C, Tan W, Zhao L, Yu D, Lin $\mathrm{D}$ and Wang L: Association of TGF- $\beta 1$ and XPD polymorphisms with severe acute radiation-induced esophageal toxicity in locally advanced lung cancer patients treated with radiotherapy. Radiother Oncol 97(1): 19-25, 2010.

18 Singh VK, Newman VL, Romaine PL, Hauer-Jensen M and Pollard HB: Use of biomarkers for assessing radiation injury and efficacy of countermeasures. Expert Rev Mol Diagn 16(1): 6581, 2016. 
19 Mazzone PJ, Wang XF, Beukemann M, Zhang Q, Seeley M, Mohney R, Holt T and Pappan KL: Metabolite profiles of the serum of patients with non-small cell carcinoma. J Thorac Oncol 11(1): 72-78, 2016.

20 Wen T, Gao L, Wen Z, Wu C, Tan CS, Toh WZ and Ong CN: Exploratory investigation of plasma metabolomics in human lung adenocarcinoma. Mol Biosyst 9(9): 2370-2378, 2013.

21 Onal C, Kotek A, Unal B, Arslan G, Yavuz A, Topkan E and Yavuz M: Plasma citrulline levels predict intestinal toxicity in patients treated with pelvic radiotherapy. Acta Oncol 50(8): 1167-1174, 2011.

22 Topkan E, Yavuz MN, Onal C and Yavuz AA: Prevention of acute radiation-induced esophagitis with glutamine in non-small cell lung cancer patients treated with radiotherapy: Evaluation of clinical and dosimetric parameters. Lung Cancer 63(3): 393 399,2009
23 Algara M, Rodríguez N, Viñals P, Lacruz M, Foro P, Reig A, Quera J, Lozano J, Fernández-Velilla E, Membrive I, Dengra J and Sanz X: Prevention of radiochemotherapy-induced esophagitis with glutamine: Results of a pilot study. Int J Radiat Oncol Biol Phys 69(2): 342-349, 2007.
Received November 16, 2016

Revised December 14, 2016

Accepted December 16, 2016 